\title{
The Effect of Backward-facing Step Height on Instability Growth and Breakdown in Swept Wing Boundary-Layer Transition
}

\author{
Jenna L. Eppink* \\ NASA Langley Research Center, Hampton, VA 23681 \\ Richard W. Wlezien ${ }^{\dagger}$ \\ Iowa State University, Ames, IA, 50011 \\ Rudolph A. King; Meelan Choudhari ${ }^{\ddagger}$ \\ NASA Langley Research Center, Hampton, VA 23681
}

\begin{abstract}
A low-speed experiment was performed on a swept flat plate model with an imposed pressure gradient to determine the effect of a backward-facing step on transition in a stationary-crossflow dominated flow. Detailed hot-wire boundary-layer measurements were performed for three backward-facing step heights of approximately 36,45 , and $49 \%$ of the boundary-layer thickness at the step. These step heights correspond to a subcritical, nearlycritical, and critical case. Three leading-edge roughness configurations were tested to determine the effect of stationary-crossflow amplitude on transition. The step caused a local increase in amplitude of the stationary crossflow for the two larger step height cases, but farther downstream the amplitude decreased and remained below the baseline amplitude. The smallest step caused a slight local decrease in amplitude of the primary stationary crossflow mode, but the amplitude collapsed back to the baseline case far downstream of the step. The effect of the step on the amplitude of the primary crossflow mode increased with step height, however, the stationary crossflow amplitudes remained low and thus, stationary crossflow was not solely responsible for transition. Unsteady disturbances were present downstream of the step for all three step heights, and the amplitudes increased with increasing step height. The only exception is that the lower frequency (traveling crossflowlike) disturbance was not present in the lowest step height case. Positive and negative spikes in instantaneous velocity began to occur for the two larger step height cases and then grew in number and amplitude downstream of reattachment, eventually leading to transition. The number and amplitude of spikes varied depending on the step height and crossflow amplitude. Despite the low amplitude of the disturbances in the intermediate step height case, breakdown began to occur intermittently and the flow underwent a long transition region.
\end{abstract}

\section{Nomenclature}

$\begin{array}{ll}c & \text { chord length, m } \\ C_{p} & \text { pressure coefficient, } C_{p}=\frac{p-p_{\infty}}{\frac{1}{2} \rho U_{\infty}^{2}} \\ f & \text { frequency, } \mathrm{Hz} \\ h & \text { step height, } \mathrm{mm} \\ N & N \text {-factor }\end{array}$

${ }^{*}$ Research Aerospace Engineer, Flow Physics and Control Branch, M.S. 170, AIAA Member

${ }^{\dagger}$ Professor and Vance and Arlene Coffman Endowed Department Chair in Aerospace Engineering, 2271 Howe Hall, Room 1200A, AIAA Fellow.

${ }^{\ddagger}$ Research Aerospace Engineer, Computational Aerosciences Branch, M.S. 128, AIAA Associate Fellow 


$R e^{\prime}$
$R e_{k}$
$T u$
$U$
$u, v, w$
$u^{\prime}, v^{\prime}, w^{\prime}$
$U_{r m s}^{\prime}$
$u_{r m s}^{\prime}, v_{r m s}^{\prime}, w_{r m s}^{\prime}$
$U_{e}$
$U_{\infty}$
$x$
$x_{c}$
$x_{h}$
$x_{r}$
$x_{s h}$
$y$
$z$
$S y m b o l s$
$\Delta N$
$\delta$

unit Reynolds number, $1 / \mathrm{m}$

Reynolds number based on excrescence height and velocity at the excrescence height turbulence intensity

mean boundary layer velocity, $\mathrm{m} / \mathrm{s}$

velocity components in the $x, y$, and $z_{n}$ directions, $\mathrm{m} / \mathrm{s}$

fluctuating components of velocity, $\mathrm{m} / \mathrm{s}$

spanwise root mean square of steady disturbance velocity, $U^{\prime}, \mathrm{m} / \mathrm{s}$

temporal root mean square of $u^{\prime}, v^{\prime}, w^{\prime}, \mathrm{m} / \mathrm{s}$

boundary layer edge velocity, $\mathrm{m} / \mathrm{s}$

freestream velocity, $\mathrm{m} / \mathrm{s}$

streamwise direction

direction normal to the leading edge

streamwise location of step

reattachment location in terms of the number of step heights downstream of the step

number of step heights downstream of step

wall-normal direction

spanwise direction (parallel to the leading edge)

shift in $N$-factor due to the excrescence

boundary layer thickness, $\mathrm{mm}$

\section{Introduction}

T AmINAR flow control remains a promising technique for improving the fuel efficiency of aircraft in the near Luture. While much progress is being made towards achieving laminar flow on wings in the laboratory, putting this into practice presents additional difficulties. Aircraft wings will have unavoidable manufacturing defects, such as steps and gaps, and necessary screws and rivets on the surface of the wing. Thus, there will be two-dimensional and three-dimensional surface irregularities on the wing that are typically avoided in wind tunnel models. It is important to understand the influence of different types of surface non-uniformities on boundary-layer transition so that appropriate manufacturing tolerances can be specified for wing designs with laminar-flow technology. Historically, the effects of 2D and 3D excrescences on transition have been predicted using an empirical method known as the $R e_{k}$ criterion. ${ }^{1-5}$ Here, $R e_{k}$ is defined as the Reynolds number based on the height of the excrescence and the undisturbed streamwise velocity at this roughness height. The $R e_{k}$ criterion correlates the measured onset of transition with a critical value of $R e_{k}$, such that the transition location first begins to move upstream from the transition location over a smooth surface as $R e_{k}$ increases above this critical value. Below this critical Reynolds number, the transition location is generally unaffected by the presence of the roughness. Recent work includes experimental and numerical studies on the effects of surface excrescences on the stability of the boundary-layer disturbances. ${ }^{6-8}$ It is assumed that a localized perturbation of the pressure distribution, caused by the surface imperfection, will cause a localized perturbation of the amplification characteristics of linear instability modes. Downstream of this perturbation, the amplification curve will become parallel to the nominal amplification curve. Hence, linear stability theory is applied to determine a $\Delta N$, that is, the change in the logarithmic amplification ratio (i.e., the so-called $\mathrm{N}$-factor) of the instabilities due to the presence of the excrescence. The majority of this work has focused on two-dimensional excrescences in two-dimensional (TS-dominated) boundary layers.

There is considerably less data available for the effect of $2 \mathrm{D}$ excrescences in $3 \mathrm{D}$ boundary layers. Perraud and Seraudie ${ }^{8}$ performed a systematic experimental study of 2D steps on a swept wing with multiple sweep angles. For the larger (i.e., more negative) angle of attack (which resulted in a more favorable pressure gradient), the increased sweep caused the boundary layer to become more sensitive to the forward-facing step height (i.e., transition occurred for lower step heights as the sweep was increased). The results for a backward-facing step showed a similar trend, although the critical roughness height Reynolds numbers were even lower. Recently, Duncan et al. ${ }^{9}$ performed flight experiments to determine the effect of $2 \mathrm{D}$ steps on transition over a swept wing. Their results indicate that the addition of the crossflow instability caused the transition location to move forward relative to the two-dimensional case, in agreement with the results of Perraud and Seraudie. ${ }^{8}$ In the companion wind tunnel testing performed by Duncan et al., ${ }^{10}$ results indicate that the 3D (swept) case is slightly more sensitive than the corresponding $2 \mathrm{D}$ (unswept) case to forward- 
facing steps, but not backward-facing steps. They also performed hot-wire measurements downstream of the step for forward- and backward-facing steps to determine the effect of the steps on stationary crossflow instabilities. They found that the steps caused an increase in N-factor for the stationary crossflow, however, the stationary crossflow amplitudes were very low at the step, and therefore the uncertainty was high. More recently, Crawford et al. ${ }^{11}$ examined the effect of step location on transition for two step locations in flight and in wind tunnel tests. It was found that, in addition to causing a critical behavior similar to that seen at the $15 \% x / c$ step location, the step at $1 \% x / c$ apparently acted as a receptivity source for the stationary crossflow, causing a gradual upstream movement of the transition front with increasing step height in the "sub-critical" regime.

Eppink et al. ${ }^{12}$ acquired detailed hot-wire measurements downstream of a backward-facing step in a stationary crossflow-dominated flow on a swept flat plate with a pressure body. The results indicated a local increase in stationary crossflow amplitude downstream of the step. Interestingly, the effect on the smaller-wavelength stationary crossflow (harmonic) mode, was much larger than the primary mode. Detailed measurements of the unsteady instabilities downstream of the step were presented and it was determined that the effect of the stationary crossflow on transition was seen through the modulation of the unsteady instabilities. Additionally, three distinct families of unsteady disturbances were identified downstream of the step corresponding to a traveling-crossflow type disturbance, a Tollmien-Schlichting instability, and a shear-layer instability. Balakumar et al. ${ }^{13}$ recently performed linear parabolized stability equation (PSE) and DNS computations for a supersonic swept-wing case to study the effect of $2 \mathrm{D}$ excrescences on stationary crossflow. Their results indicate a linear effect on the growth of the stationary crossflow modes, but the different modes were affected differently. The shorter wavelength modes were amplified due to the step, but the longer wavelength modes were actually stabilized relative to the baseline case.

There is clearly a gap in knowledge concerning the details of how transition is affected on a swept wing when two-dimensional excrescences are introduced. The above-mentioned research in this area has been concerned primarily with studying the effect of forward- and backward-facing steps on the transition location. There is very little data that can provide an understanding of how the steps actually affect the boundary-layer instabilities and how this ultimately leads to transition. In order to gain more insight into this problem, an experiment was performed to enable a detailed look at the effect of a backward-facing step on transition in an otherwise stationary crossflow-dominated swept-wing flow. Detailed results were presented previously by Eppink et al. ${ }^{12}$ for a single backward-facing step height. The current work is a follow-on to this work, focusing on the effect of step height on the disturbance growth and taking a closer look at the breakdown process. Detailed boundary layer measurements are presented for three different step heights. The effect of step height on stationary crossflow growth is discussed in Section III.A. Section III.B covers the effect of step height on the unsteady disturbance growth and breakdown, and Section III.C discusses the effect of stationary crossflow amplitude on breakdown.

\section{Experimental Setup}

The experiment was performed in the 2 Foot by 3 Foot Low Speed Boundary Layer Channel at NASA Langley Research Center. The tunnel is a closed circuit facility with a $0.61 \mathrm{~m}$ high by $0.91 \mathrm{~m}$ wide by 6.1 $\mathrm{m}$ long test section. The tunnel can reach speeds up to $45 \mathrm{~m} / \mathrm{s}\left(R e^{\prime}=2.87 \times 10^{6} / \mathrm{m}\right)$ in the test section.

Freestream turbulence intensity levels, $T u=\frac{1}{U_{\infty}} \sqrt{\frac{1}{3}\left(u^{\prime 2}+v^{\prime 2}+w^{\prime 2}\right)}$, were measured using a crosswire in an empty test section to be less than $0.06 \%$ for the entire speed range of the tunnel, and less than $0.05 \%$ for the test speed of $26.5 \mathrm{~m} / \mathrm{s}$. This value represents the total energy across the spectrum (from $0.25 \mathrm{~Hz}$ to 10 $\mathrm{kHz}$ ), and has not been filtered to remove the low-frequency acoustic component. Thus, this tunnel can be considered a low-disturbance facility for purposes of conducting transition experiments. ${ }^{14}$

The $0.0127-\mathrm{m}$ thick flat plate model consists of a $0.41 \mathrm{~m}$ long leading-edge piece, swept at $30^{\circ}$, and a larger downstream piece (see Fig. 1). The model is $0.91 \mathrm{~m}$ wide (thus, spanning the width of the test section) and $2.54 \mathrm{~m}$ long on the longest edge. The downstream or leading-edge pieces can be adjusted relative to each other using precision shims to create either forward-facing or backward-facing 2D steps of different heights, parallel with the leading edge. The leading-edge piece was polished to a surface finish of $0.2 \mu \mathrm{m}$, and the larger downstream plate had a surface finish of $0.4 \mu \mathrm{m}$. The chord, $c$, is taken as the longest edge of the plate $(2.54 \mathrm{~m})$ and is used to non-dimensionalize quantities throughout the paper. Thus, the step is located at $x / c=0.161$. A leading-edge contour was designed for the bottom side of the plate to make the suction-peak less severe, and therefore avoid separation, which could potentially cause unsteadiness in the 
attachment line.

A 3D pressure body along the ceiling was designed to induce a streamwise pressure gradient so that transition would be dominated by stationary crossflow. The ceiling liner was contoured to simulate nearly infinite swept-wing flow within a mid-span measurement region of width 0.3 meters. This was achieved by designing the liner such that the $C_{p}$ contours were parallel with the leading edge within the core region. The ceiling liner was fabricated out of a hard foam using a computer-controlled milling machine.

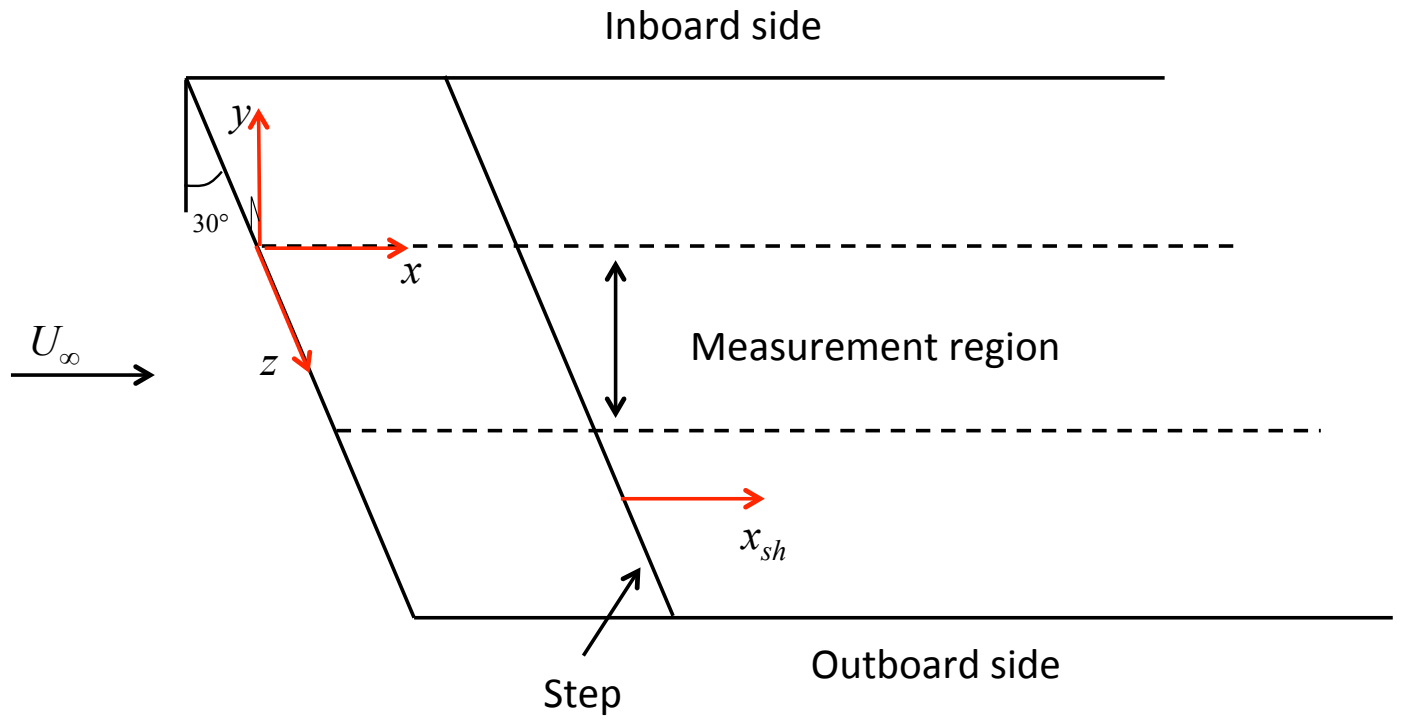

Figure 1: Coordinate system

All measurements were performed at a freestream velocity of $26.5 \mathrm{~m} / \mathrm{s}\left(R e^{\prime}=1.69 \times 10^{6} / \mathrm{m}\right)$. The data were acquired using a hot-wire mounted on a traversing system that could be moved in all $3(x, y$, and $z)$ directions. Detailed boundary-layer measurements allowed for tracking of the instability growth and determining the effect of the backward-facing step on the instabilities. Additionally, sublimating chemical flow visualization was performed using naphthalene to determine transition location. Three different leading-edge roughness configurations were investigated: a clean leading edge and two DRE (discrete roughness element) configurations. The DREs were applied with a spanwise spacing, $\lambda_{z}$, of $11 \mathrm{~mm}$ and were approximatly $20 \mu \mathrm{m}$ thick. DREs with two different diameters were used: $2.75 \mathrm{~mm}$ (small DREs) and $4.4 \mathrm{~mm}$ (large DREs). The large diameter DREs led to larger initial amplitudes of the stationary crossflow due to the enhanced receptivity. The spacing of the DREs $(11 \mathrm{~mm})$ corresponds to the most amplified stationary crossflow wavelength calculated for the baseline case with no step.

Hot-wire data were obtained using a constant temperature anemometer with a 1:1 bridge configuration. Single sensor hot-wire probes were used throughout the test for the boundary-layer measurements. The wires were $5-\mu \mathrm{m}$ diameter, platinum plated tungsten wires with a sensor length of $1.25 \mathrm{~mm}$. The data were AC-coupled at $0.25 \mathrm{~Hz}$ to acquire the fluctuating velocity component and low-pass filtered at a cut-off of 10 $\mathrm{kHz}$. Data were typically digitized at a sampling rate of $25 \mathrm{kHz}$. For more details of the experiment setup, as well as data reduction and uncertainty analysis, refer to Eppink et al. ${ }^{12}$

\section{Results and Discussion}

Detailed boundary-layer measurements were acquired for three backward-facing step (BFS) heights $(h / \delta \approx 0.36,0.45$, and 0.49$)$ and are presented in this section. The two larger step heights were chosen because they were found to bracket the critical step height (i.e., the step height above which transition abruptly moved upstream). The $h / \delta \approx 0.45$ step height was slightly lower than the critical step height, while the $h / \delta \approx 0.49$ step height was slightly larger than the critical step height. It was expected that insight 
could be gained into the dominant transition mechanisms by studying transition at these nearly critical step heights. The smallest step height of $h / \delta \approx 0.36$ was studied briefly since it was found to be completely subcritical. For this case, only one leading-edge roughness configuration was tested (small DREs). More detailed results for the baseline and largest step height cases are presented by Eppink et al., ${ }^{12}$ but some are included herein for comparison purposes.

Figure 2 shows the mean profiles at numerous streamwise stations for the $h / \delta=0.45$ step case with large DREs, along with computations from the baseline case. These computations were performed using the experimental mid-span $C_{p}$ distribution from the baseline case, under the assumption of an infinite span swept airfoil. See Eppink et al. ${ }^{12}$ for more details of the computation. The profiles separate downstream of the step. Reattachment occurs by around 30 step heights, and the profiles return to the unperturbed state by approximately 50-55 step heights downstream of the step. Overall, the experimental results match well with the boundary-layer profiles computed using the measured $C_{p}$ distribution in the mid-span region, although there is some discrepancy near the wall for some of the profiles. The discrepancy could be due to the distortion of the profiles due to the stationary crossflow (since the experimental profiles are taken only at one spanwise station).

Approximate transition locations for each step height are tabulated in Table 1. These results are based on the naphthalene flow visualization results for the no DRE case for each step height. The transition location is averaged over the outboard half of the measurement region. Results are given in terms of $x / c$ and in terms of $x_{s h}$ for comparison with later results.

Table 1: Backward-facing step heights and approximate transition locations for no DRE cases

\begin{tabular}{|c|c|c|c|}
\hline Case & Step Height $(h / \delta)$ & Transition location $(x / c)$ & Transition location $\left(x_{s h}\right)$ \\
\hline Baseline & 0 & 0.48 & $\mathrm{x}$ \\
Small & 0.36 & 0.48 & 722 \\
Intermediate & 0.45 & 0.48 & 929 \\
Large & 0.49 & 0.27 & 340 \\
\hline
\end{tabular}
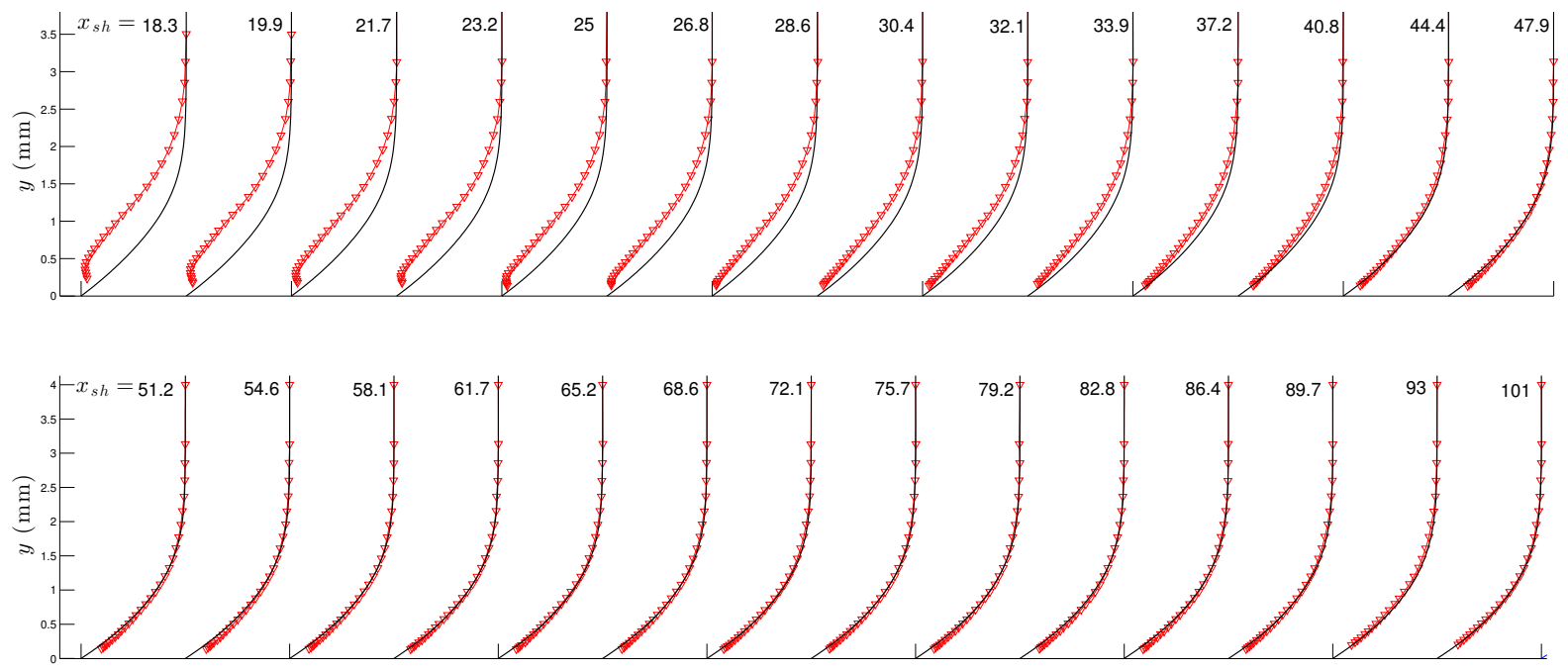

Figure 2: Measured mean profiles (symbols) for $h / \delta=0.45$ BFS large DRE case compared with baseline computation (solid lines) 


\section{A. Effect of Step Height on Stationary Crossflow Growth}

The growth of the stationary crossflow instability is examined in Figs. 3 and 4 for all of the configurations tested. The amplitudes are plotted along with the $N$-factor plots for the primary mode associated with the DRE spacing $\left(\lambda_{z}=11 \mathrm{~mm}\right)$ and the harmonic $\left(\lambda_{z}=5.5 \mathrm{~mm}\right)$. The amplitudes were calculated by integrating the wavelength spectra over a range of wavelengths around the primary and harmonic modes. The ranges used for each mode are $\lambda_{z}=8$ to $20 \mathrm{~mm}$ for the primary mode and $\lambda_{z}=5$ to $8 \mathrm{~mm}$ for the harmonic.

The stationary crossflow amplitudes at the most upstream measurement station $(x / c \approx 0.12)$ for the baseline (no-step) cases with DREs were used as the initial amplitudes for the experimental $N$-factors. The most upstream measurement location for the no-step, no-DRE case was farther downstream at $x / c=0.18$, and hence, the initial amplitude was chosen such that the $N$-factor at $x / c=0.18$ matched the $N$-factors for the two DRE cases at this location. The initial amplitudes for the largest step case were equivalent to the initial amplitudes used for the baseline case. A new batch of appliques was used for the DREs for the second phase of measurements involving the two smaller step heights. Unfortunately, this second batch of DREs was found to be slightly thicker than the previous batch (approximately 16\% thicker). Thus, it is expected that the initial amplitudes for the two DRE cases would be larger than those during the earlier measurements for the large step case. The initial amplitudes used for this second batch of DREs was found using the procedure described above for the baseline case (i.e., using the amplitude at $x / c=0.12$ if available and, if not, then by matching the $N$-factor at the most upstream station with that of the baseline case), and indeed the initial amplitudes were found to be larger by approximately $25 \%$.

As seen in Fig. 3, the step has a local effect on the growth of the stationary crossflow, causing a local increase in amplitude (for most cases) that increases with step height. The exception is the smallest step, which appears to cause a local decrease in amplitude of the primary mode. The $N$-factors near the step for the two DRE cases agree well with each other for each step height. No measurements were acquired near the step for the no DRE case due to the small amplitude of the stationary crossflow at the step. The maximum $\Delta N$ values, calculated as the maximum deviation from the baseline $N$-factors, are given in Table 2 for each of the DRE cases and wavelengths. The larger step causes a much larger $\Delta N$ for the primary mode that is over 7 times the $\Delta N$ for the intermediate step case. However, the max $\Delta N$ for the harmonic does not change much between the two larger step heights, but the $\Delta N$ of the harmonic for the smallest step height is approximately half of that for the two larger step heights. The max $\Delta N$ for all step heights is much higher for the harmonic than for the primary mode, although the actual amplitude of the harmonic mode is much lower.

Table 2: Max $\Delta N$ values

\begin{tabular}{|c|c|c|}
\hline Step Height $(h / \delta)$ & $\lambda_{z}=8$ to $20 \mathrm{~mm}$ & $\lambda_{z}=5$ to $8 \mathrm{~mm}$ \\
\hline 0.36 & -0.465 & 0.890 \\
0.45 & 0.113 & 1.775 \\
0.49 & 0.818 & 1.732 \\
\hline
\end{tabular}

There appears to be a fairly significant reduction in stationary crossflow amplitude downstream of the step for the small and large DRE cases for the intermediate step height. No such effect is seen for the no DRE case. Balakumar et al. ${ }^{13}$ observed a decrease in the linear growth of stationary crossflow downstream of a backward-facing step. However, the reduction in amplitude seen in the present work is probably not a linear effect, but is likely due to the flow beginning to undergo breakdown. Breakdown to turbulence occurs shortly downstream of the step for the large step height case. The flow downstream of the intermediate step does not become fully turbulent until much farther downstream. However, as will be discussed in depth in the next section, the flow starts to intermittently breakdown starting shortly downstream of the step. The reduction in amplitude is probably due to the fact that breakdown extends over a very long region. Thus, the stationary crossflow amplitude is attenuated. It is still possible that this could result in a delay in transition (i.e., the location of fully turbulent flow) - it essentially becomes a balancing act between the stationary crossflow and the unsteady disturbances.

The smallest step height was studied in order to study this apparent reduction in amplitude to determine if it is a linear effect of the step on the stationary crossflow or if it is an effect of the flow starting to undergo breakdown. As seen in Fig. 3, the small step apparently causes a local decrease in amplitude close to the 
step, but the amplitude soon collapses back to that of the baseline case. Thus, it appears that the reduction in stationary crossflow amplitude far downstream of the step in the larger step cases is due to the flow beginning to undergo breakdown, which causes an attenuation of the stationary crossflow.

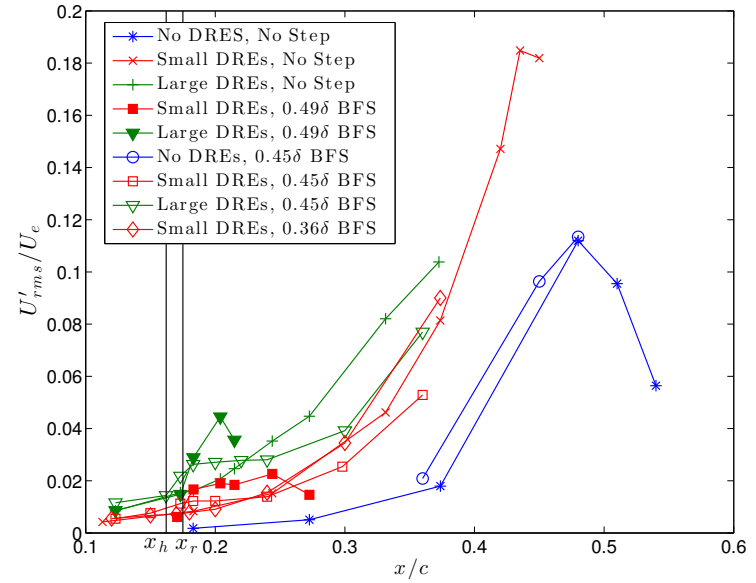

(a) Amplitude evolution of stationary crossflow $\left(\lambda_{z}=8\right.$ to $20 \mathrm{~mm}$ )

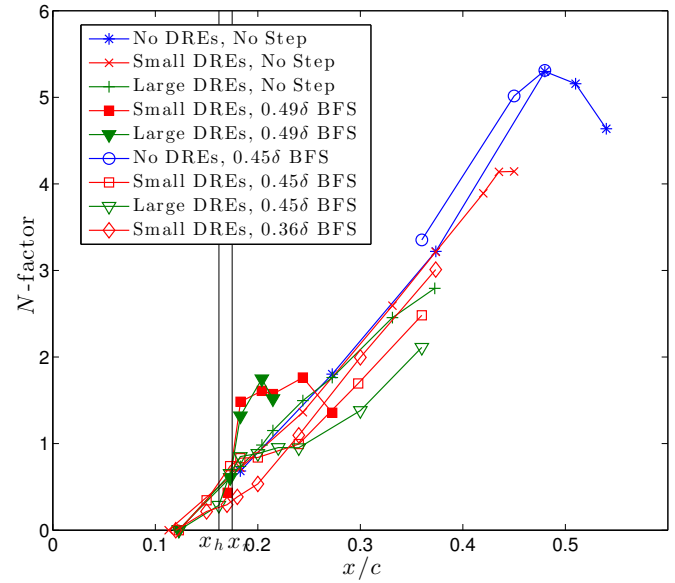

(b) Growth of $11 \mathrm{~mm}$ stationary crossflow mode

Figure 3: Amplitudes and $N$-factors of primary stationary crossflow mode. Step $\left(x_{h}\right)$ and reattachment $\left(x_{r}\right)$ locations are indicated by the black vertical lines.

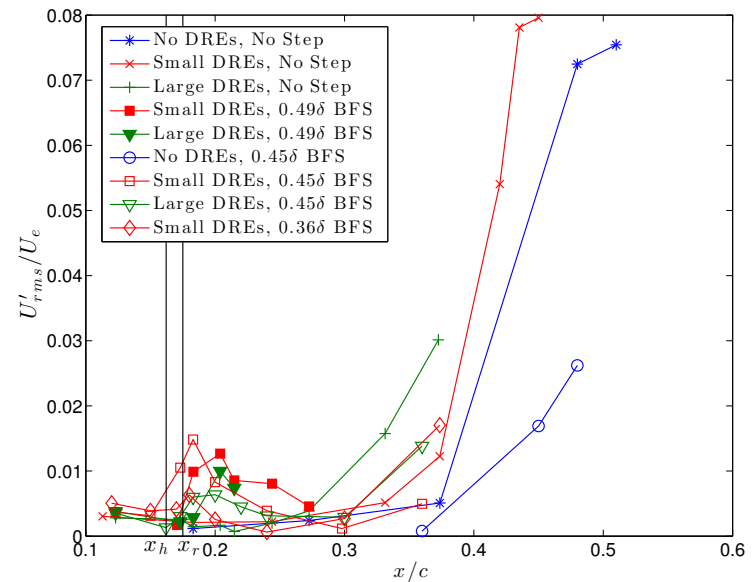

(a) Amplitude evolution of stationary crossflow $\left(\lambda_{z}=5\right.$ to $8 \mathrm{~mm}$ )

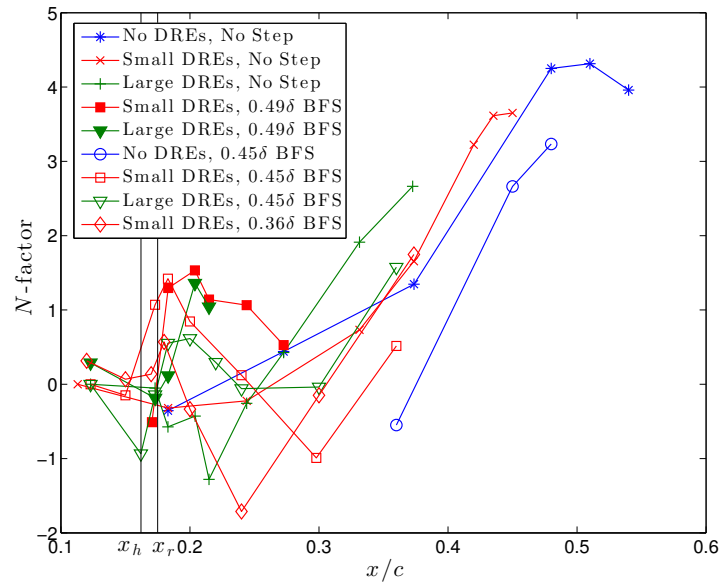

(b) Growth of $5.5 \mathrm{~mm}$ stationary crossflow mode

Figure 4: Amplitudes and $N$-factors of harmonic stationary crossflow mode. Step $\left(x_{h}\right)$ and reattachment $\left(x_{r}\right)$ locations are indicated by the black vertical lines.

\section{B. Effect of Step Height on Unsteady Disturbance Growth and Breakdown}

Results for the largest step height case are discussed in detail in Eppink et al., ${ }^{12}$ but will be summarized briefly here as context for the following discussion of the other step height results. The velocity spectra downstream of the step were found to be rich with unsteady disturbances in a broad frequency band ( $f \approx 80$ to $1500 \mathrm{~Hz}$ ). These unsteady disturbances were not present in the no-step case, and are believed to be directly responsible for transition with the step present since the stationary crossflow amplitudes remained low at transition. 
Data were acquired simultaneously from two hot-wires in the boundary layer to extract phase speed and wave angle information. Three distinct disturbance bands were identified based on these results corresponding to a traveling crossflow type disturbance $(f=80$ to $200 \mathrm{~Hz})$, a TS-like disturbance $(f=200$ to $800 \mathrm{~Hz})$, and a shear-layer disturbance $(f=800$ to $1500 \mathrm{~Hz})$. These frequency bands will henceforth be referred to as the Lband, M-band, and H-band for low, mid, and high, respectively. The unsteady disturbances were modulated in the spanwise direction by the stationary crossflow, forming regions of peaks and valleys in amplitude with spanwise spacing corresponding to the dominant stationary crossflow wavelength. This is evident in Fig. 5a for the M-band in the intermediate step case. The initial amplitude of the stationary crossflow affected the peak amplitudes of the unsteady disturbances, causing them to increase with increasing initial amplitude, and thus causing transition to move upstream. Therefore, the effect of stationary crossflow on transition in the backward-facing step case is understood to be due to the modulation of the unsteady disturbances. For clarity, the discussion in the remainder of this section will focus on the small DRE case for all three step heights.

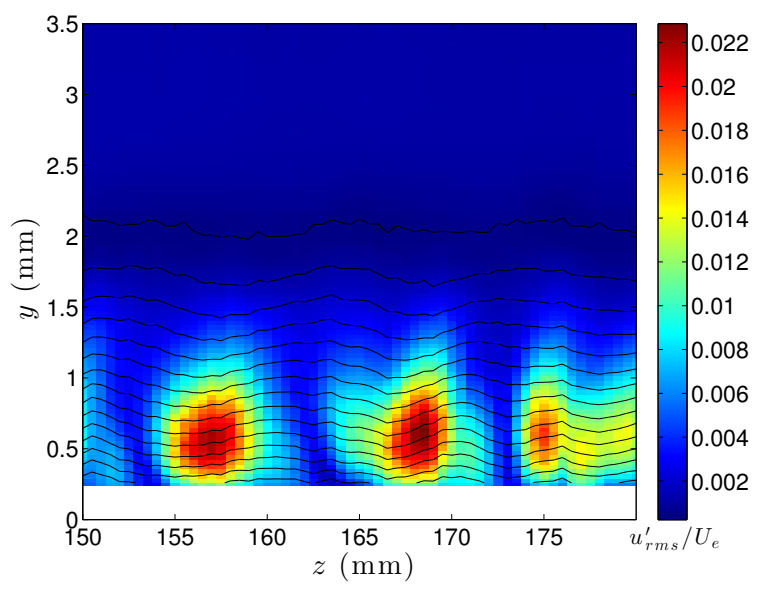

(a) $u_{r m s}^{\prime}$ for M-band

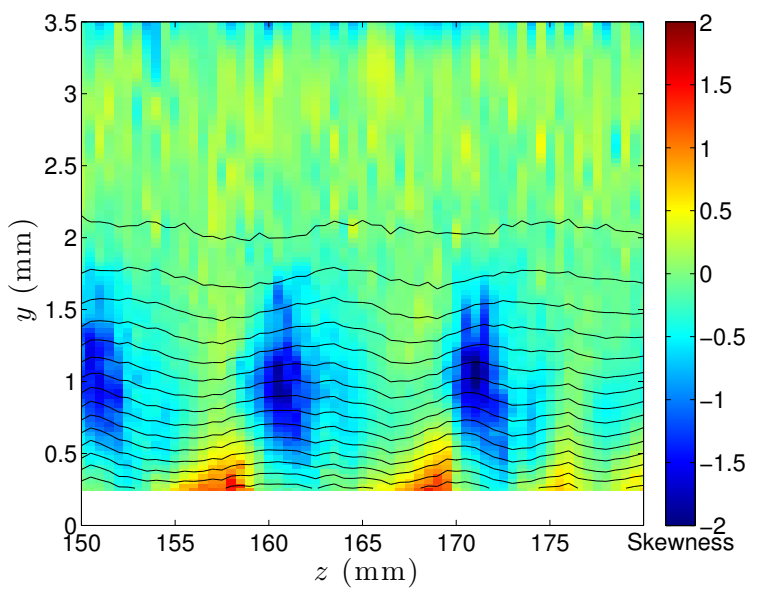

(b) Skewness

Figure 5: $u_{r m s}^{\prime}$ and skewness contours at $x_{s h}=75$ for $h / \delta=0.45$ BFS, large DREs. Solid black lines are mean flow contours.

The streamwise evolution of the three disturbance bands was studied by choosing a spanwise peak location of the $u_{r m s}^{\prime}$ amplitude (for instance $z=157 \mathrm{~mm}$ in Fig. 5a), and tracking that peak location downstream. Single boundary layer profiles were acquired at the peak location for numerous streamwise stations. The peak location was chosen for the M-band since the distortion of the M- and H-bands were similar, and thus, the spanwise peak locations were similar. The L-band distortion was somewhat different from the M- and H-bands, and the peak location was typically offset from the M-band by a few millimeters. Therefore, while the measured amplitudes are probably not the absolute peak of the L-band disturbance, the observed trends should still hold. Throughout the boundary layer scans, spanwise scans were performed to verify the peak location of the M-band.

The results from this approach for each of the disturbance bands and for all step heights are shown in Fig. 6. The unsteady disturbance amplitudes in this figure are taken from the maximum amplitude of each $u_{r m s}^{\prime}$ boundary-layer profile. Based on the largest step height results, it was conjectured that below the critical step height, the unsteady disturbances should still grow but peak at some low amplitude and then decay. The intermediate step height does result in significantly lower amplitudes for all three disturbance bands when compared to the largest step height. In fact, the M-band peaks in amplitude close to 100 step heights downstream of the step, at an amplitude of less than $2 \%$ of $U_{e}$. The $\mathrm{H}$-band stays below $0.1 \% U_{e}$ in amplitude until $x_{s h} \approx 170$. The L-band also levels off in amplitude at around $x_{s h}=150$ at approximately $1 \% U_{e}$. However, it is interesting that breakdown appears to still be triggered (albeit much slower) for this lower step height. Note that the growth in energy in all three frequency bands at this intermediate step height that starts at approximately $x_{s h}=200$ is no longer due to growth of the actual modes themselves but is instead indicative of the beginning of the breakdown process and occurs due to spectral broadening. 


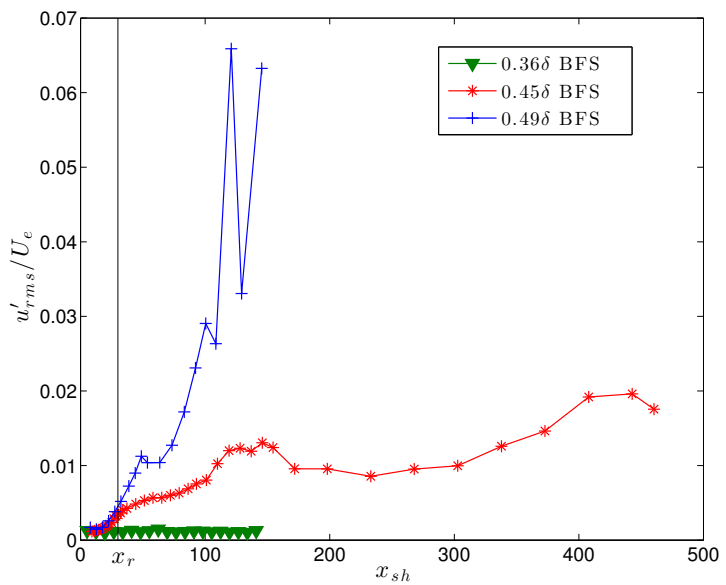

(a) L-band

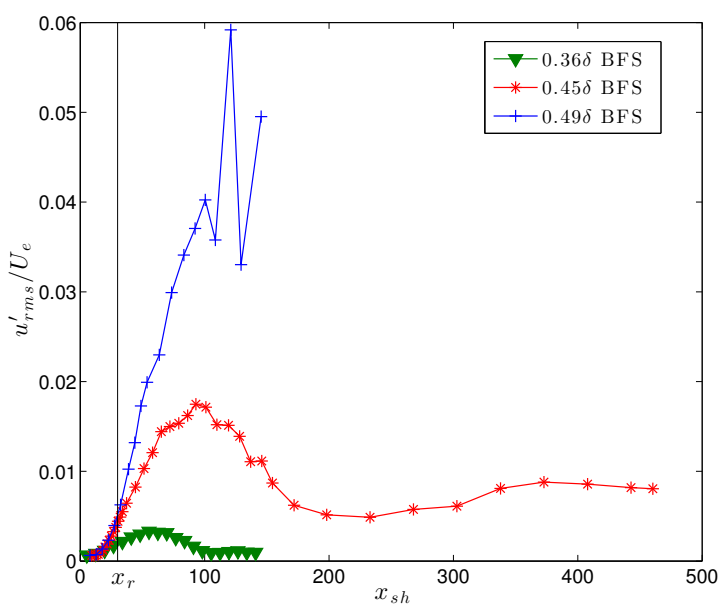

(b) M-band

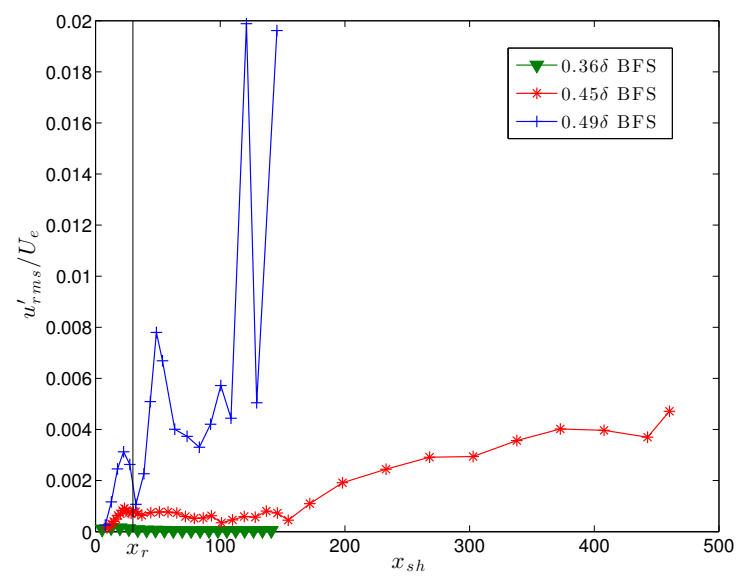

(c) H-band

Figure 6: Peak unsteady disturbance growth along a streamline, small DREs.
For the large and intermediate step height cases, the H-band experiences a short region of growth in the separated region, followed by a short region of decay, followed by another region of growth just downstream of reattachment. The disturbance peaks in amplitude approximately 50 step heights downstream of the step, and subsequently decays. In the largest step height case, this second region of growth is quite significant, however, the amplitudes do not even reach $1 \% U_{e}$ before the disturbance decays again.

This second region of growth is associated with a drastic change in wave angle of the $\mathrm{H}$-band that occurs just downstream of reattachment. The disturbance changes from a nearly streamwise-traveling wave to a nearly spanwise-traveling wave, similar to the L-band. Due to this change in behavior, the fact that the growth occurs downstream of reattachment (which would not be expected for a simple shear-layer instability), and an observed increase in bicoherence between the L- and $\mathrm{H}-$ bands, this second region of growth of the H-band is believed to be due to nonlinear interactions with the Lband.

The lowest step height case results in growth of the $\mathrm{M}$ - and H-bands, and, as expected, the peak amplitudes are much lower than in the two larger step cases. The M-band peaks at an amplitude of $0.3 \%$ of $U_{e}$, while the $\mathrm{H}$-band hardly grows above the noise floor, peaking at $0.02 \% U_{e}$ at 12 step heights downstream of the step before decaying. There is no visible growth in the L-band frequency range for this case.

Breakdown for both of the larger step height cases was preceded by the appearance of large spikes in velocity. A time trace showing this phenomenon is shown in Fig. 7, along with a figure zoomed in on a single spike. This figure is included to show that the spikes themselves are not high frequency, such as the spikes seen by Klebanoff et al. ${ }^{15}$ in the fundamental breakdown of TS waves on a flat plate. The spike shown in Fig. 7b has a period corresponding to a frequency of about $500 \mathrm{~Hz}$.

Similar to Klebanoff's results, positive and negative spikes were found to occur at different spanwise and wallnormal locations and seem to be spatially related to the spanwise modulation of the unsteady disturbances and, thus, to the modulation of the mean flow, as illustrated in Fig. 5b. The skewness is plotted in this figure as a way of showing the spatial locations of the positive and negative spikes. The positive spikes (associated with a positive skewness factor) typically appear near the wall, while the negative spikes occur off of the wall and offset in the spanwise direction from the location of the positive spikes. Klebanoff et al. ${ }^{15}$ conjectured that the spikes that they observed were due to the shedding of hairpin eddies. It is not clear whether these spikes are related to a similar mechanism, but they are believed to be due to some type of breakdown mechanism that is triggered 
by the unsteady disturbances.

The number of positive peaks in the time trace greater than a chosen threshold level was counted as a way of quantifying and comparing the number of velocity spikes occurring for each case. The threshold level was based on the standard deviation of the fluctuating velocity, rather than just a set amplitude. This approach is slightly more robust since the unsteady disturbances vary in amplitude depending on the location. A level of five times the standard deviation was chosen as the threshold for the values plotted in Fig. 8a. The number of spikes in the time trace is counted at each point in the boundary layer, and then the maximum number of spikes in the boundary layer is plotted for that streamwise station. Similarly, Fig. 8b shows the maximum amplitude of the spikes at each streamwise location. Note that these results are taken at a single spanwise point in a highly three-dimensional flow, thus, the results at a given streamwise station do not necessarily represent the behavior across the span. However, general trends can still be identified and put into context based on the limited number of $2 \mathrm{D}$ boundary layer scans that were performed.

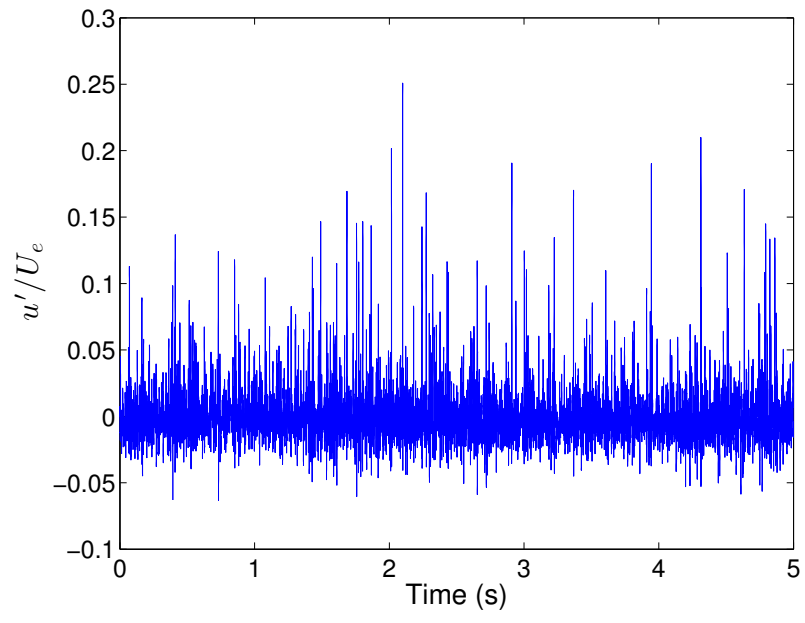

(a) Time trace example

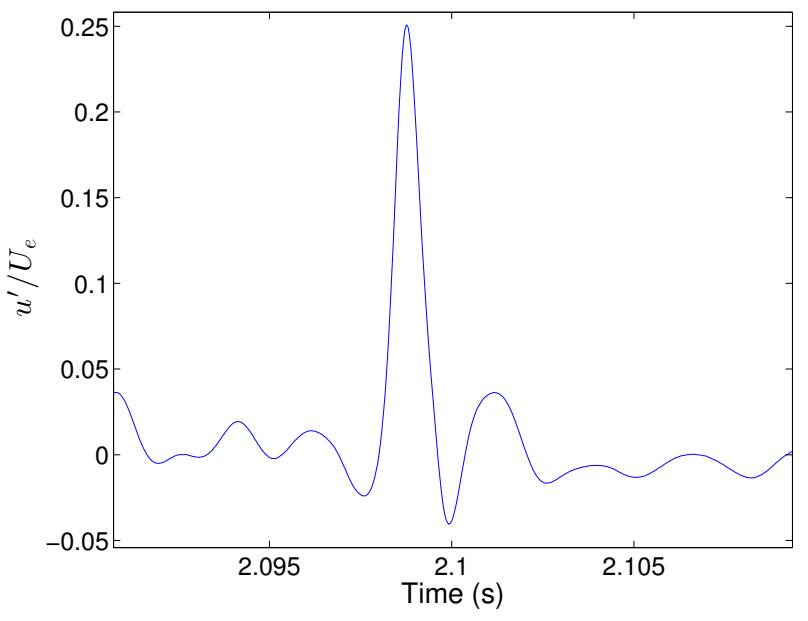

(b) Time trace zoomed

Figure 7: Time trace showing numerous positive spikes.

Positive spikes are actually first observed in the separated region downstream of the step for all three step cases, although they are very small in amplitude. The number of spikes decreases again near reattachment, but then begins to increase again at approximately $x_{s h}=50$ for the intermediate step. Interestingly, the streamwise location at which the spikes begin increasing in number and amplitude is similar (though maybe 5-10 step heights farther upstream) for the largest step height case compared to the intermediate step height. The major difference appears to be in the number and amplitude of the spikes as the flow continues downstream. For the largest step height, the spikes increase in number rapidly moving downstream, while the spike count increases much slower for the intermediate step height case. Despite the disturbance levels remaining low for the intermediate step height case, the spikes still appear intermittently and continue downstream, leading to a rather long transitional region. In fact, the number of positive spikes actually reaches a maximum at a similar location to where the M-band amplitude peaks $\left(x_{s h} \approx 100\right)$, and then subsequently decreases, but the peak amplitude of the spikes continues to increase downstream. Almost no spikes are present in the smallest step height case, though some do occur just downstream of the step in the separated region, similar to the other cases.

A similar process is repeated for the negative spikes. These results are shown in Figs. 9a and 9b. No negative spikes are evident in the separated region. There is a short region for the largest step case in which the number of negative spikes increases drastically before decreasing quickly. This region corresponds to the nonlinear growth region identified earlier for the H-band, and this behavior is not evident in the positive spike count. Thus, it appears that for the largest step case, the negative spikes are somehow related to the growth of the H-band downstream of reattachment. None of the other step cases exhibit this sudden increase in the number of negative spikes, nor do they exhibit such a large growth of the H-band disturbance downstream of reattachment (see Fig. 6c). Given this, the H-band may be what causes earlier transition in the largest step case, despite the fact that it is of lower amplitude compared to the other disturbances 
present.

Other than this short region just downstream of reattachment, the negative spike count in general is lower than the positive spike count for a given location and case. Similarly, the maximum negative spike amplitudes are somewhat lower than the maximum positive spike amplitudes. As noted previously, these results were acquired at a single spanwise station for each streamwise location and thus do not give a full picture of the flow field. In fact, as mentioned earlier, the positive and negative spikes were observed to occur offset from each other in span (as illustrated by the skewness contour plot in Fig. 5b). The spanwise location at which these data were taken corresponds to a spanwise location close to where the positive spikes would be expected to be most prominent. Thus, it is expected that, at this location, the negative spikes would occur less frequently and be of lower amplitude than the positive spikes.

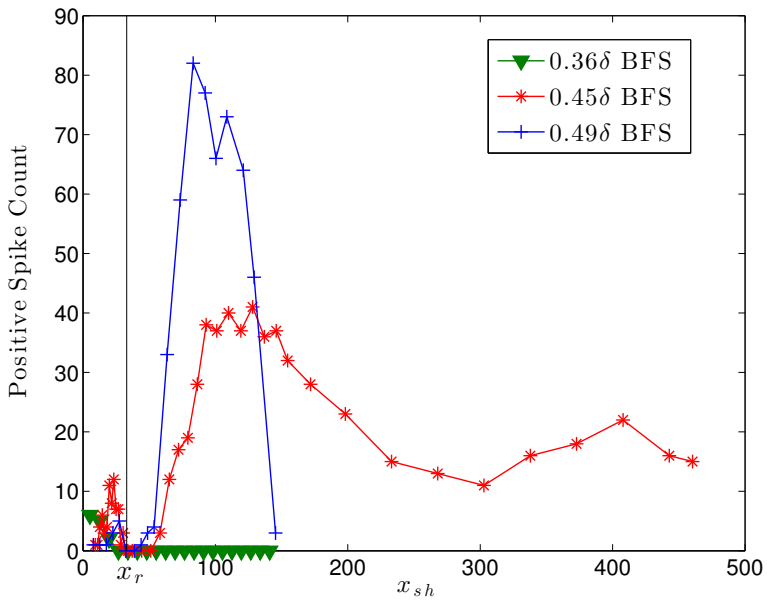

(a) Maximum number of positive spikes from each profile

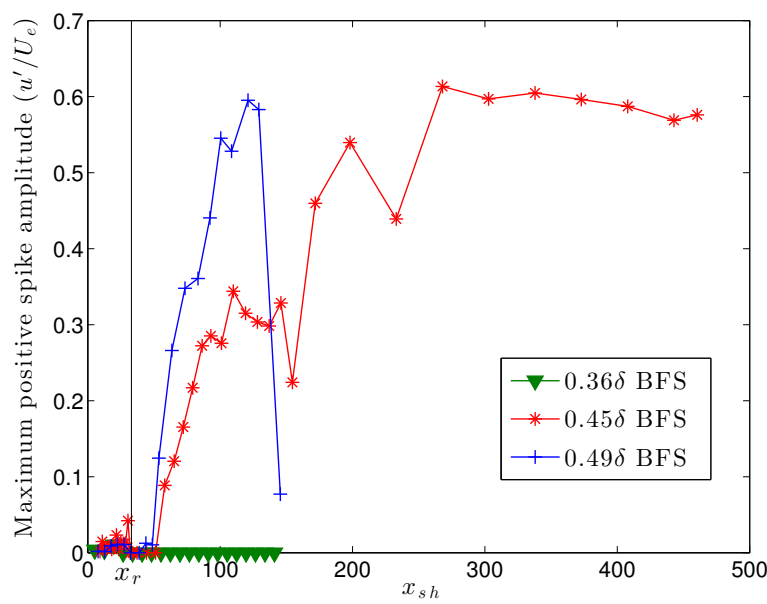

(b) Maximum spike amplitude from each profile

Figure 8: Streamwise evolution of positive spikes, small DREs, all 3 step heights.

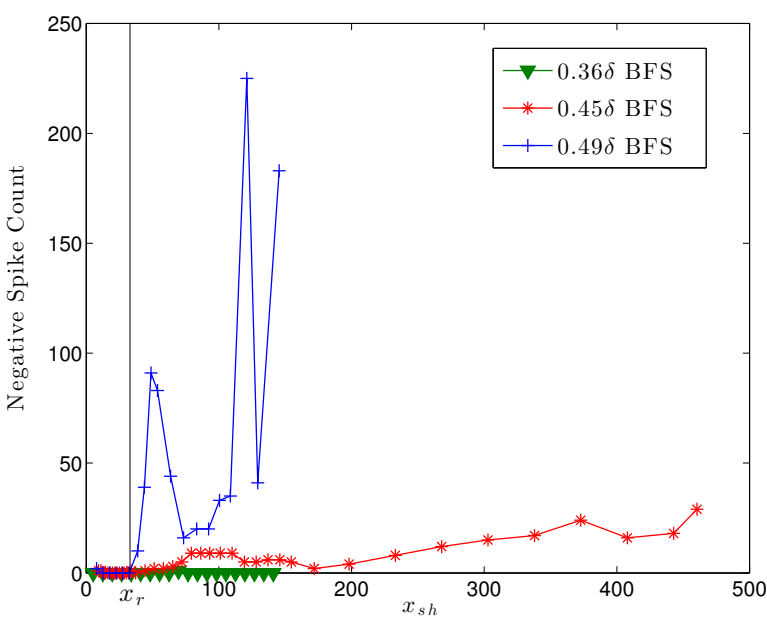

(a) Maximum number of negative spikes from each profile

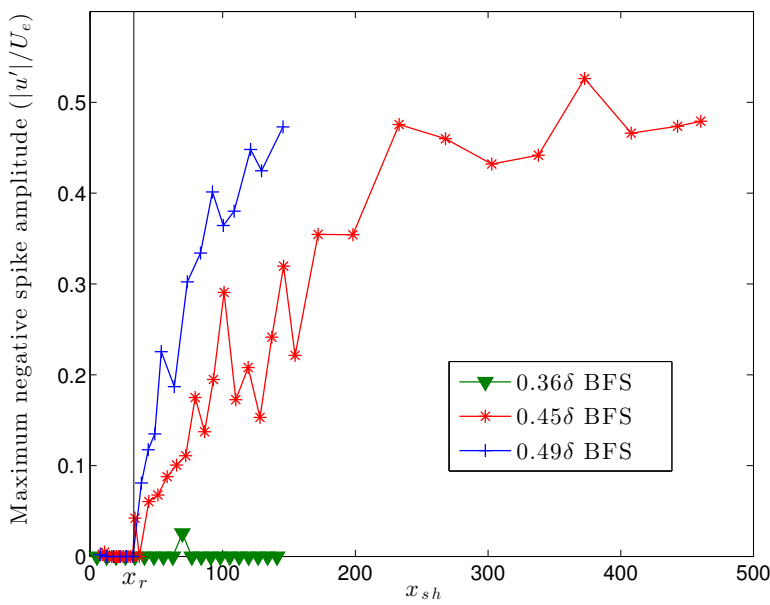

(b) Maximum negative spike amplitude from each profile

Figure 9: Streamwise evolution of negative spikes, small DREs, all 3 step heights.

Eventually, the spikes become very large in amplitude $\left(\geq 0.3 U_{e}\right)$, and begin to undergo breakdown. To illustrate the breakdown evolution, a plot of intermittency vs. $x_{s h}$ is shown in Fig. 10. The intermittency is computed for each time trace by first high-pass filtering the data at a cutoff frequency of 6250 . The high-pass 
filtered data is then analyzed by computing the RMS at each time using a moving window. A threshold RMS level is chosen by manual comparison of the time trace data to the computed RMS values. The threshold was chosen to be 5 times the baseline (laminar flow) value of the computed RMS. The intermittency at that point is then computed as the fraction of time above the threshold. The maximum intermittency in each boundary layer profile is plotted in Fig. 10. The intermittency increases drastically starting at approximately $x_{s h}=90$ in the large step height case. In the intermediate step case, the intermittency rises gradually, beginning at approximately $x_{s h}=120$. The main difference between the large and intermediate step height is the length of the breakdown region. The spikes occurring in the intermediate step height case are extremely intermittent, and thus, the transition region is much longer than for the larger step.

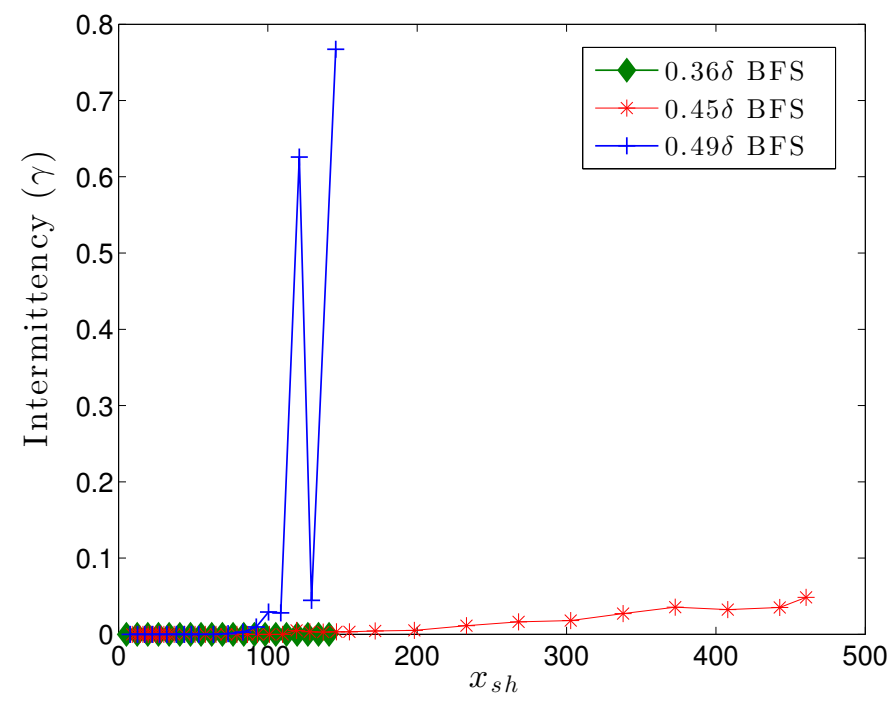

Figure 10: Intermittency vs. $x_{s h}$, small DREs, all 3 step heights.

\section{Effect of Stationary Crossflow Amplitude on Breakdown}

As was mentioned briefly in the previous section, the initial stationary crossflow amplitude was found to have an effect on the amplitude of the unsteady disturbances, as illustrated in Fig. 11 for the intermediate and large step heights. It can be clearly seen that as the stationary crossflow amplitude is increased, the growth of the unsteady disturbances increases as well, particularly downstream of reattachment. This section briefly explores how breakdown is affected by this phenomenon.

The positive spike count and maximum spike amplitude are shown vs. $x_{s h}$ for the intermediate and large step cases in Figs. 12a and 13a, respectively. There is a clear pattern relating to the stationary crossflow initial amplitude (i.e., the different DRE cases). As the initial stationary crossflow amplitude is increased, the number of spikes increases more quickly moving downstream. This same behavior is observed for the peak amplitude, the exception being the small and large DRE cases in the intermediate step case, which have almost identical maximum spike amplitudes throughout the measurement domain. This relationship between stationary crossflow amplitude and the appearance and amplitude of the spikes is believed to be related to the fact that the unsteady disturbance peak amplitude increases with increasing stationary crossflow amplitude, as seen in Fig. 11. Thus, the occurrence of the spikes is believed to be related to the amplitude of the unsteady disturbances, though it could also be related to the increased modulation of the mean flow due to the increased stationary crossflow amplitude. With the available data, it is not possible to separate the two effects.

The evolution of intermittency vs. $x_{s h}$ is shown in Fig. 14 for the intermediate and large step cases. Note the different y-axis scales in these figures. The increase in stationary crossflow initial amplitude clearly causes a more rapid rise in intermittency for both step cases. Thus, the increase in number and amplitude of the spikes caused (either directly or indirectly) by the increase in stationary crossflow amplitude leads to earlier and faster breakdown. The difference is much more drastic in the larger step height case than for the intermediate step case. 


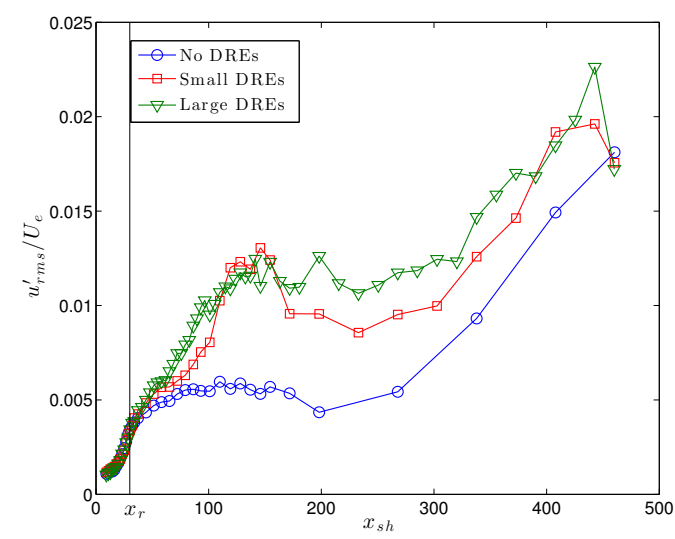

(a) L-band, intermediate step

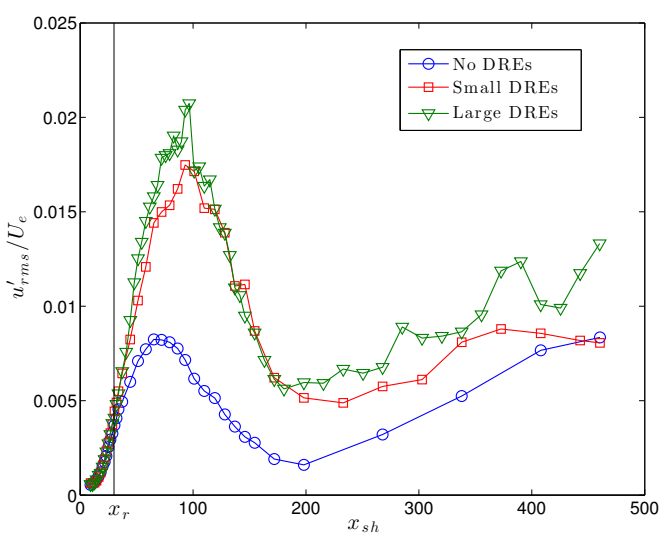

(c) M-band, intermediate step

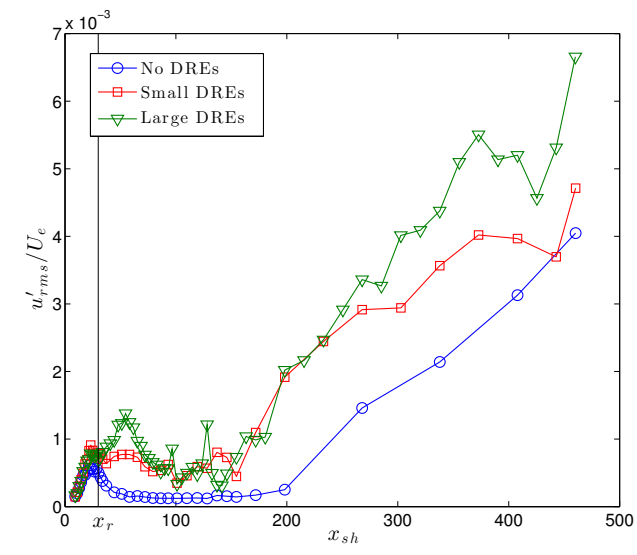

(e) H-band, intermediate step

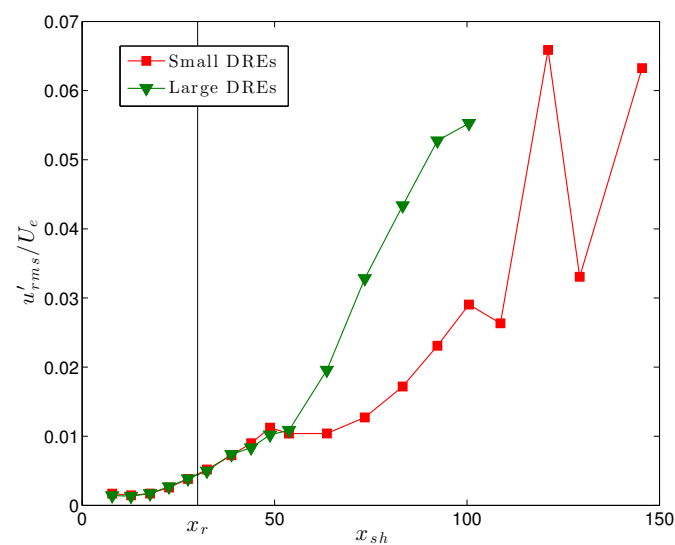

(b) L-band, large step

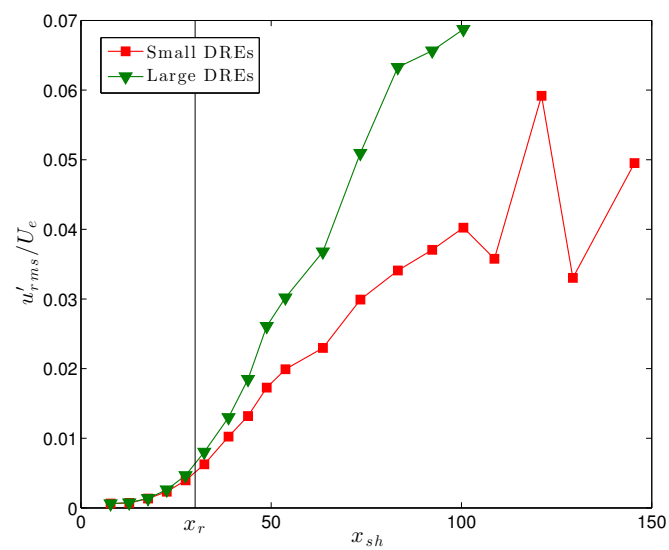

(d) M-band, large step

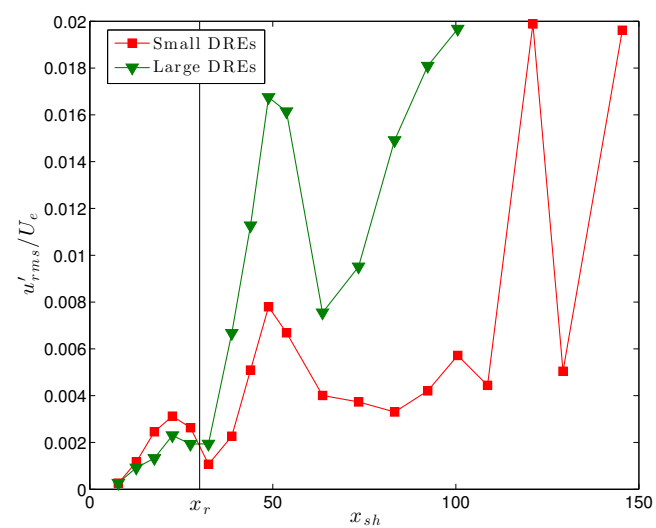

(f) H-band, large step

Figure 11: $u_{r m s}^{\prime}$ vs. $x_{s h}$ for intermediate and large step heights. 


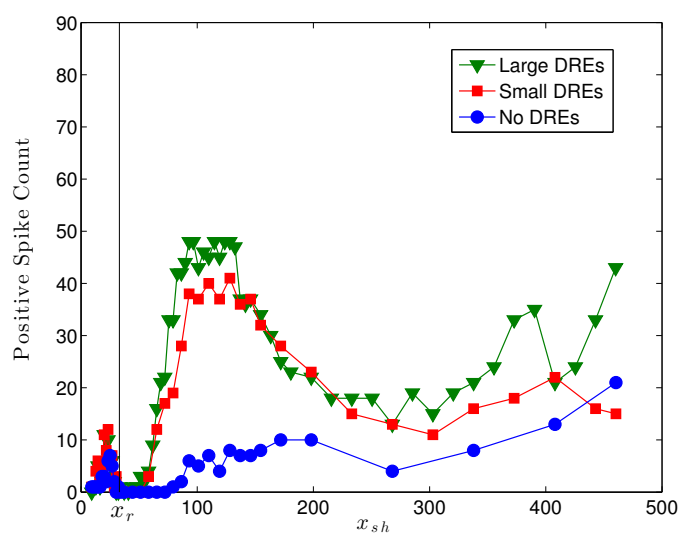

(a) Positive spike count

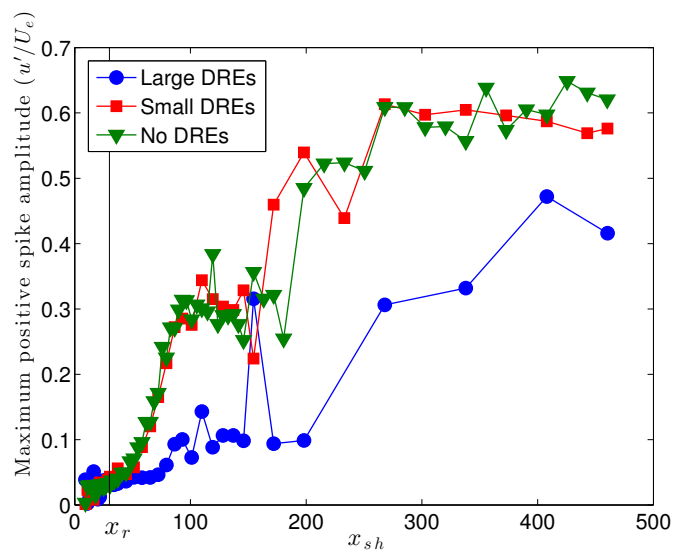

(b) Positive spike maximum amplitude

Figure 12: Streamwise evolution of positive spikes for intermediate step case.

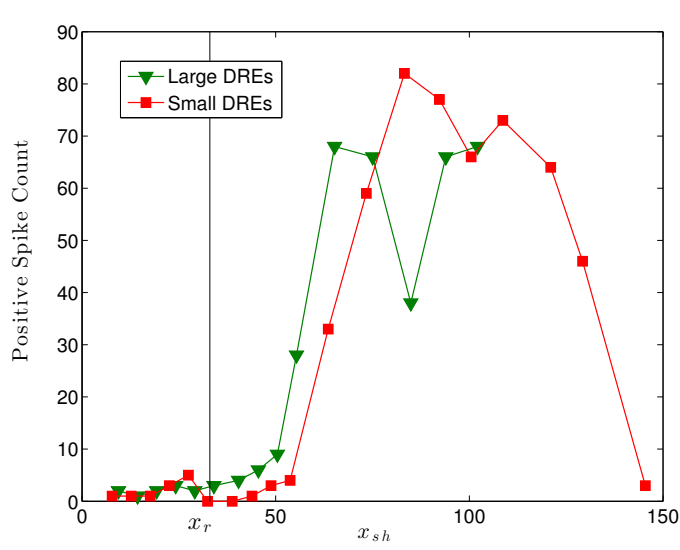

(a) Positive spike count

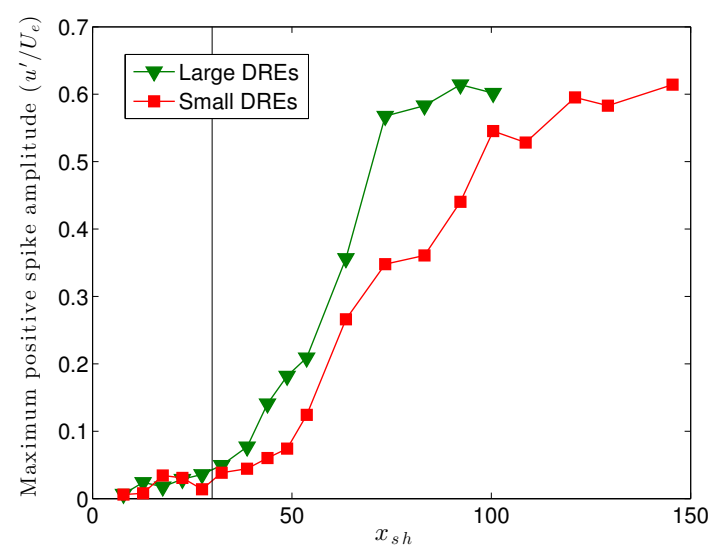

(b) Positive spike maximum amplitude

Figure 13: Streamwise evolution of positive spikes for large step case.

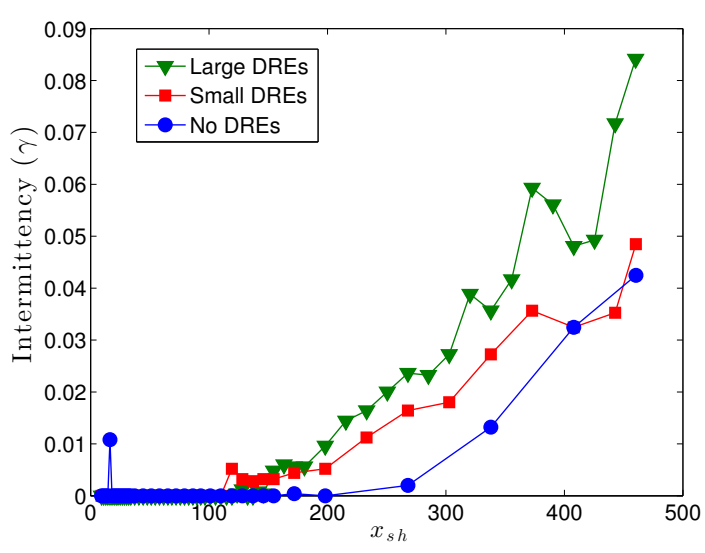

(a) Intermediate step

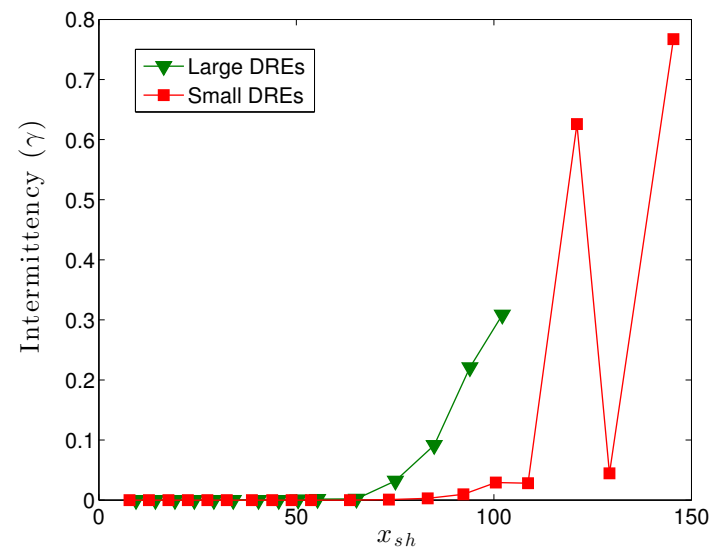

(b) Large step

Figure 14: Intermittency vs $x_{s h}$ for both step cases. 


\section{Summary and Concluding Remarks}

Detailed boundary layer measurements were performed for three different backward-facing step heights and three different stationary crossflow initial amplitude cases in a stationary crossflow-dominated flow. Stationary crossflow growth was investigated for the primary and harmonic modes and was compared with the baseline results. The stationary crossflow was locally affected by the step but did not directly lead to transition in the critical case. However, stationary crossflow was still found to be important in the transition process with the step present. Even at very low amplitudes $\left(1-2 \% U_{e}\right)$, stationary crossflow causes modulation of the unsteady disturbances, thereby introducing three-dimensionality, which is known to be important for the breakdown process.

The growth of the unsteady disturbances was also studied in detail by tracking the peak of the disturbance. The amplitudes decreased with decreasing step height and with decreasing initial stationary crossflow amplitude. At the lowest step height $(0.36 h / \delta)$, the L-band was not observed. At the intermediate step height $(0.45 \mathrm{~h} / \delta)$, the amplitudes of the L- and M-bands peaked between 1-2\% $U_{e}$ before decaying, and the H-band was an order of magnitude lower in amplitude. In the largest step height case, the L- and M-bands grew much faster than in the smaller step cases, reaching amplitudes of approximately $7 \% U_{e}$ by $x_{s h} \approx 100$. The H-band grew initially in the separated region, then decayed and grew again downstream of reattachment, peaking at around $x_{s h}=50$. It is not unexpected that the unsteady disturbance amplitude should decrease with decreasing step height, though it is interesting that the L-band was not at all present in the smallest step case. What is somewhat unexpected is that breakdown was triggered in the intermediate step case even though all the disturbances involved remained low in amplitude.

Breakdown (i.e., the first appearance of intermittent flow) was preceded by the appearance of large velocity spikes in the two larger step height cases. No spikes were observed in the smallest step height case. The spikes occurred locally, with positive spikes occurring near the wall and the negative spikes occurring away from the wall and offset from the positive spikes in the span. Depending on the step height and initial stationary crossflow amplitude, the spikes increased in number and amplitude starting downstream of the reattachment location. Eventually, the spikes reached large amplitudes and began to undergo breakdown. The spikes were extremely intermittent in the intermediate step case. Thus, if one considers the beginning of transition to be the location at which a single spike starts to exhibit high frequency breakdown (spectral broadening), then the transition region was much longer for the intermediate step height case compared to the larger step height.

The spikes are believed to be a manifestation of some type of breakdown mechanism due to the interaction of the stationary crossflow vortices and unsteady disturbances, though the exact mechanism is not yet clear. It may be somewhat similar to the K-type breakdown mechanism as observed by Klebanoff et al., ${ }^{15}$ but in this case the stationary crossflow vortices play the role of creating the spanwise variation in the unsteady disturbance amplitude. Some similarities exist in the hot-wire measurements, specifically the locations of the positive spikes (near the wall) and negative spikes (away from the the wall and offset in the span). There is, however, one large difference in the observed phenomenon - namely the period of the spikes in this case are similar to the period of the primary modes (i.e., the unsteady disturbances), not an order of magnitude higher as seen by Klebanoff.

Some important conclusions can be drawn from this work. The first conclusion is that, at least in this scenario, (i.e., backward-facing step downstream of the neutral point), "bypass transition" is not the cause of transition at the critical step height. Bypass, in this context, refers to the sudden (somewhat mysterious) transition to turbulence which bypasses the growth of any primary disturbance. In fact, there is some step height below the "critical" height (as observed by surface flow-visualization) at which the transition mechanism differs from the baseline case. Spikes begin to appear shortly downstream of the step, but depending on the conditions, can persist over a long distance before fully turbulent flow develops.

A second conclusion is that the stationary crossflow vortices (and thus, sweep) play a role in this critical behavior because the stationary vortices promote the three-dimensionality in the unsteady flow field. It is believed that the interaction of the stationary crossflow vortices and the unsteady disturbances leads to the appearance of these spikes and early breakdown. The spanwise spacing of the positive and negative spikes corresponds to the stationary crossflow spanwise wavelength, and thus, the spike locations are also related to the locations of the peaks and valleys of the unsteady disturbances (caused by the interaction with the stationary crossflow vortices). It is also interesting to note that this breakdown mechanism begins to occur when the disturbance amplitudes are still very low $\left(1-2 \% U_{e}\right)$. Further work is being performed to try to gain a better understanding of this breakdown mechanism and how it is triggered. A better understanding of what 
triggers transition in these swept-wing excrescence cases will allow for higher-fidelity transition prediction.

\section{Acknowledgments}

This work was performed as part of the Revolutionary Computational Aerosciences discipline under the Transformational Tools and Technologies Project of NASA's Fundamental Aeronautics Program. The authors would like to thank William T. Jones for his assistance with the ceiling contour design, Jackson Johnson for fabrication of the ceiling liner, Charlie Debro for his support of the wind tunnel testing, and the members of the Flow Physics and Control Branch of NASA Langley Research Center for their support and many helpful discussions.

\section{References}

\footnotetext{
${ }^{1}$ Holmes, B., Obara, C., Martin, G., and Domack, C., "Manufacturing tolerances for natural laminar flow airframe surfaces," SAE Paper 850863, 1985.

${ }^{2}$ Fage, A., "The smallest size of a spanwise surface corrugation which affects boundary layer transition on an aerofoil," R.\&M. No. 2120, British A.R.C., 1943.

${ }^{3}$ Carmichael, B., "Surface waviness criteria for swept and unswept laminar suction wings," Norair Report No. NOR-59-438 (BLC-123), 1959.

${ }^{4}$ Sedney, R., "A survey of the effects of small protuberances on boundary-layer flows," AIAA Journal, Vol. 11, 1973, pp. $782-792$.

${ }^{5}$ Tani, I., "Boundary-layer transition," Annual Review of Fluid Mechanics, Vol. 1, No. 1, 1969, pp. 169-196.

${ }^{6}$ Crouch, J., Kosorygin, V., and Ng, L., "Modeling the effects of steps on boundary-layer transition," IUTAM Symposium on Laminar-Turbulent Transition, Springer, 2006, pp. 37-44.

${ }^{7}$ Wang, Y. and Gaster, M., "Effect of surface steps on boundary layer transition," Experiments in Fluids, Vol. 39, No. 4, 2005, pp. 679-686.

${ }^{8}$ Perraud, J. and Seraudie, A., "Effects of steps and gaps on 2D and 3D transition," European Congress on Comp. Methods in Applied Science and Eng., ECCOMAS, 2000, pp. 11-14.

${ }^{9}$ Duncan Jr, G., Crawford, B., and Saric, W., "Effects of Step Excrescences on Swept-Wing Transition," AIAA Paper 2013-2412, 2013.

${ }^{10}$ Duncan Jr, G. T., Crawford, B. K., Tufts, M. W., Saric, W. S., and Reed, H. L., "Effects of Step Excrescences on a Swept Wing in a Low-Disturbance Wind Tunnel," AIAA Paper 2014-0910, 2014.

${ }^{11}$ Crawford, B. K., Duncan, G. T., Tufts, M. W., Saric, W. S., and Reed, H. L., "Effects of Step-Excrescence Location on Swept-Wing Transition," AIAA Paper 2013-2412, 2013.

${ }^{12}$ Eppink, J., Wlezien, R., King, R., and Choudhari, M., "The Interaction of Stationary Crossflow and a Backward-facing Step in Swept Boundary-Layer Transition," AIAA Paper 2015-0273, 2015.

${ }^{13}$ Balakumar, P., King, R. A., and Eppink, J. L., "Effects of Forward-and Backward-Facing Steps on the Crossflow Receptivity and Stability in Supersonic Boundary Layers," AIAA Paper 2014-2639, 2014.

${ }^{14}$ Saric, W. S. and Reshotko, E., "Review of flow quality issues in wind tunnel testing," AIAA Paper 1998-2613, 1998.

${ }^{15}$ Klebanoff, P., Tidstrom, K., and Sargent, L., "The three-dimensional nature of boundary-layer instability," J. Fluid Mech, Vol. 12, No. 1, 1962, pp. 1-34.
} 